\title{
Investigation of the Relations between Biogenic and Anthropogenic Sources of Organic Atmospheric Aerosol under Conditions of Restrictions Due to the Coronavirus Pandemic
}

\author{
G. G. Dultseva ${ }^{a, *}$ and S. N. Dubtsov ${ }^{a, * *}$ \\ ${ }^{a}$ Voevodsky Institute of Chemical Kinetics and Combustion, Siberian Branch, Russian Academy of Sciences, \\ Novosibirsk, 630090 Russia \\ *e-mail:dultseva@kinetics.nsc.ru \\ **e-mail:dubtsov@kinetics.nsc.ru
}

Received March 26, 2021; revised March 26, 2021; accepted April 13, 2021

\begin{abstract}
The effect of restrictions caused by the COVID-19 pandemic in Novosibirsk in spring 2020 on the composition of trace atmospheric gases and organic aerosol in the air of Novosibirsk Akademgorodok is investigated. The concentrations of aldehydes (formaldehyde, acetaldehyde, benzaldehyde, acrolein, and substituted aromatic aldehydes), the corresponding carboxylic acids, and peroxy compounds were studied before and after the adoption of restrictions, prior to the start of growing season, that is, with the minimal emission of biogenic compounds into the atmosphere. Differences in diurnal variations and the composition of hydroperoxides are revealed on a background of weakening anthropogenic sources. The role of hydroperoxides in gas-to-particle conversion is shown. In parallel, the concentrations of nanometer-sized aerosol particles were measured, and differences in their chemical composition were analyzed. To explain the observed changes in the composition of organic aerosol, the stages formation of condensable products was numerically simulated. A sharp decrease in the strength of anthropogenic sources allowed us to reveal and characterize the contribution from biogenic sources to the formation of organic atmospheric aerosol and to describe this process.
\end{abstract}

Keywords: atmospheric organic aerosol, formaldehyde, acetaldehyde, aromatic aldehydes, aerosol formation DOI: $10.1134 / \mathrm{S} 1024856021050067$

\section{INTRODUCTION}

Restrictions adopted in many countries in spring 2020 due to the coronavirus pandemic caused a general decrease in the concentrations of gaseous pollutants and solid particles in the surface air. An improvement of air quality was observed even in satellite images [1]. The effect of restrictions on air quality was studied in the United States, Great Britain, China, India, and other countries [1-4]. Almost all those studies discovered that a decrease in the concentrations of gaseous organic substances in the air was not as substantial as had been expected in view of a sharp decrease in industrial activity in the corresponding regions. The concentrations of organic aerosol in the surface air did not show any tendency to decrease; they even increased in some regions, though the strength of anthropogenic sources decreased by several times [3]. For this reason, the necessity arose to evaluate the changes that occurred in the relations between natural and anthropogenic sources of atmospheric organic aerosol. The conditions that had been created during restrictions, till lockdown, allowed one to exclude definite anthropogenic sources (industrial emissions, vehicle exhaust) almost completely and to evaluate the intensity of natural sources alone in the formation of organic aerosol.

The goal of our investigation was to reveal the role of biogenic sources in the formation of atmospheric organic aerosol and to establish the level of regional conventional background for gaseous compounds that are usually considered as the pollutants of the urban atmosphere (aldehydes, ketones, hydroperoxides, organic peroxides, peroxynitrites, and nitrates).

\section{MATERIALS AND METHODS}

Air was sampled in March-July 2020 on the territory of the Novosibirsk Scientific Center; meteorological parameters were recorded. Sampling was performed by pumping air with the help of an aspirator through sorption tubes filled with crushed quartz as a sorbent, with absorbing solutions $(0.5 \mathrm{~mL})$ deposited on it. To measure the concentrations of aldehydes, the tubes were filled with a solution of 2,4-dinitrophenylhydrazine acidified to $\mathrm{pH} 2$ with sulfuric acid. To determine the concentrations of organic peroxides, air 


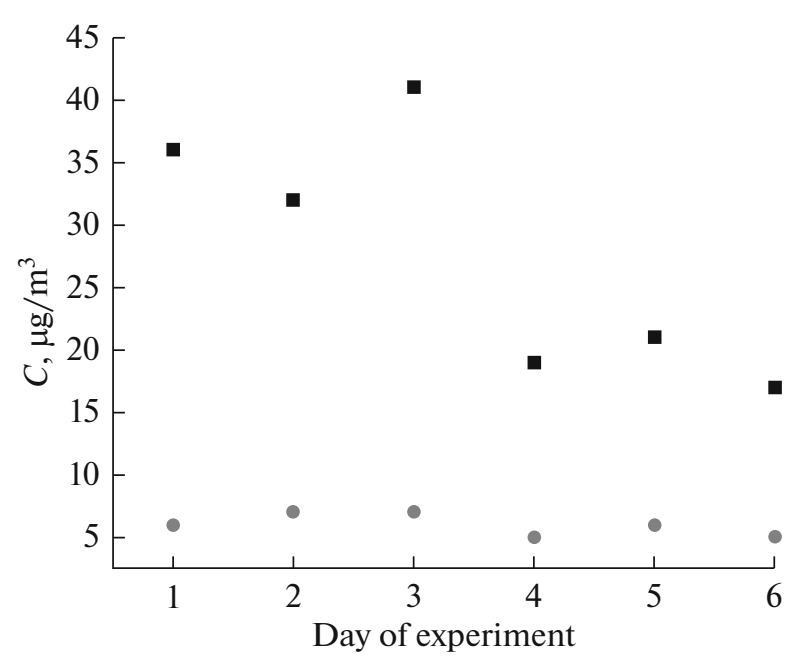

Fig. 1. Daily maxima of the concentrations of formaldehyde (squares) and acetaldehyde (circles); along the abscissa, 1, 2, 3-the days before the restrictions were imposed; 4, 5, 6-the days after the restrictions were imposed.

was pumped through absorbing vessels filled with a mixture of ethanol and butanol at a ratio of $1: 1$ by volume. The volume of the solution in absorbing vessels was $6 \mathrm{~mL}$.

The samples were analyzed by means of high-performance liquid chromatography (HPLC) with Milikhrom A-02 (Econova, Russia) equipped with a standard column $75 \mathrm{~mm}$ long. Chromatographic conditions were as follows: the column was filled with ProntoSIL 120-5-C18 AQ sorbent, the column temperature was $35^{\circ} \mathrm{C}$, eluents were acetonitrile (SigmaAldrich, $\geq 99.9 \%$ ) + distilled water (gradient from 0 to $75 \mathrm{vol} \%)$, the volume of the sample introduced into the chromatograph was $20 \mu \mathrm{L}$. The chromatograms were processed using AlfaSpectr software (v.1.0).

The concentrations of nitrogen oxides and ozone in the air were measured using standard photometric procedures [5].

The concentrations of submicron aerosol particles in the surface air were determined using a diffusion aerosol spectrometer (ICKC SB RAS). Measurements were carried out in the continuous mode with automatic data processing. Atmospheric aerosol was also sampled for chemical analysis using AFA-KhA filters, then the sampled substance was transferred into solution through ultrasonic treatment for subsequent chromatographic analysis.

Calculation of the kinetics of chemical transformations was carried out using NICK 2.2 software [6]. The rate constants of elementary stages included in the model were taken from the NIST chemical kinetics database [7]. For the stages with unknown rate constants, estimated values were used. These values were obtained by comparing bond energies in a reagent under study and in a chemically similar compound for which the reaction rate constants are known. The sensitivity of the kinetic model to the variations in input parameters was tested in special calculation procedures.

\section{RESULTS AND DISCUSSION}

The results were compared with the measurements performed in the days with similar meteorological conditions (the absence of atmospheric precipitation, fine weather, weak wind): we chose three days meeting these requirements on the week preceding the introduction of restrictions, and three days on the week after the restrictions came into force. The days were numbered sequentially.

The measurement results showed that under restrictions the concentrations of nitrogen oxides in the air sharply decreased, while ozone concentration increased beyond expectations. So, when the anthropogenic sources were switched off, nitrogen dioxide was no longer playing the role of the major source of ozone in the ground air layer $\left(\mathrm{NO}_{2}+h v \rightarrow \mathrm{NO}+\mathrm{O}\right.$, $\mathrm{O}_{2}+\mathrm{O} \rightarrow \mathrm{O}_{3}$ ). No new sources of ozone appeared in that situation, so it was evident that ozone concentration increased due to a decrease in the rate of its consumption for the oxidation of organic compounds. Below, this result is compared with the measured changes in the concentrations of peroxide compounds.

Diurnal variations in the concentrations of formaldehyde and acetaldehyde in the urban atmosphere usually have clearly pronounced maxima corresponding to the maximal traffic intensity (in the morning and in the evening). The ratio of formaldehyde to acetaldehyde concentrations characteristic of vehicle exhaust is $10: 1$, while the benzaldehyde concentration varies between 2 and $7 \%$ of the formaldehyde concentration [8]. Substituted aromatic aldehydes are not present in vehicle emissions, and the concentrations of these compounds in cities are usually small. After the restrictions were imposed, the traffic intensity decreased by a factor of 10-12 on average (determination was carried out for six days: three days before and three days after the restrictions were imposed, we calculated the vehicles moving in both directions along the road near which the observation site was located). We did not detect a proportional decrease in aldehyde concentrations for any representative of this class of compounds.

The maximal concentrations of formaldehyde and acetaldehyde during these six days are shown in Fig. 1. One can see that formaldehyde content sharply decreased, while acetaldehyde concentration remained nearly the same.

Acrolein concentration was only slightly dependent on traffic intensity but substantially increased with a northeasterly wind, which suggests that acrolein was 
transported from a remote source, the intensity of which did not change substantially. Benzaldehyde concentration was very low during the six days $\left(<2 \mu \mathrm{g} / \mathrm{m}^{3}\right)$, which is generally usual for urban air in spring. No significant changes in benzaldehyde concentration were detected. It has been shown in our previous works that aromatic aldehydes, in particular benzaldehyde, are more efficient sources of aerosol than aliphatic aldehydes: the yield of aerosol products from formaldehyde photolysis is about 100 times lower than from benzaldehyde photolysis [9]. Because of this, a sharp decrease in formaldehyde concentration does not cause a corresponding decrease in the concentration of organic atmospheric aerosol.

In the presence of aldehydes in the air, structurally related carboxylic acids are also detected. However, this regularity seemed to be violated after the restrictions were imposed: with a 2-fold decrease in formaldehyde concentration, the concentration of formic acid increased by $70 \%$, while the concentration of acetic acid decreased almost to the detection limit. The data on the concentrations of carboxylic acids in the air over the days of the experiment are shown in the Table 1.

Measurements of the concentrations of submicron particles in the air showed that the morning maximum (07:00-08:00) remained almost unchanged after the introduction of restrictions, while the afternoon maximum (16:00-18:00) decreased approximately by a factor of two. The same behavior was revealed for the total concentration of hydroperoxide compounds. Investigation of the diurnal variations of hydroperoxide concentrations showed that their maxima preceded (by approximately an hour) the maxima of the concentrations of submicron particles: 06:00-07:00 (the morning maximum) and 15:00-16:00 (the afternoon maximum).

Changes in the total content of hydroperoxides at the morning maximum during six days of observation are shown in Fig. 2. Before the introduction of restrictions, this value was $25 \mu \mathrm{g} / \mathrm{m}^{3}$ on average, while under restrictions it sharply decreased to the values below $5 \mu \mathrm{g} / \mathrm{m}^{3}$. It was demonstrated above that ozone concentration increased, so it may be assumed that the rate of consumption of hydroperoxides increased.

The analysis of the chemical composition of aerosol particles showed that restrictions caused a substantial decrease in the concentrations of oxygen- and nitrogen-containing components in the particulate matter. This allows us to assume that with an increase in ozone concentration, the contribution from competitive reactions leading to the formation of gasphase products increased, and a decrease in the content of nitrogen-containing groups in the aerosol is due to a sharp decrease in the concentration of nitrogen dioxide.

It should be noted that the measurements were carried out before the start of the growing season, when

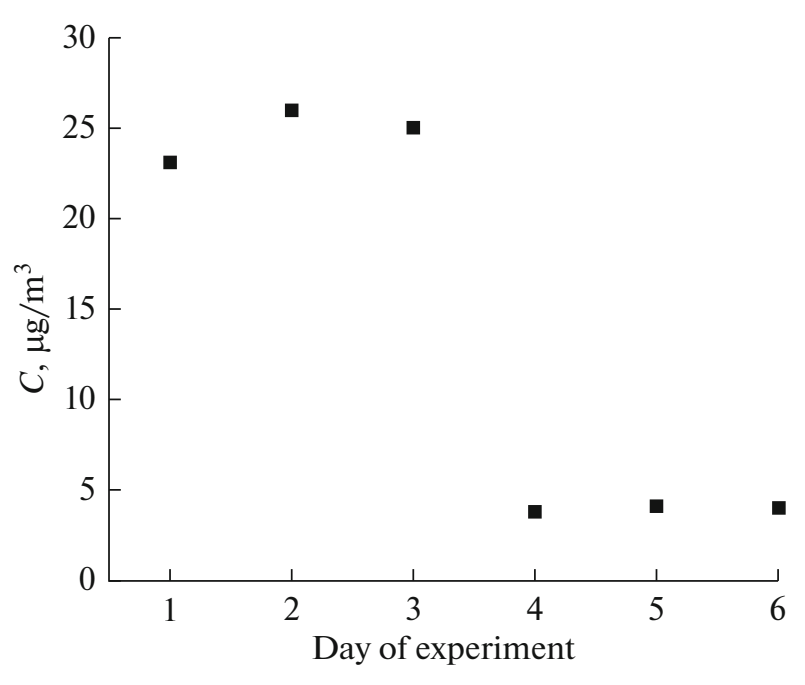

Fig. 2. Total content of hydroperoxides during the six-day experiment.

the contribution from biogenic sources into the concentrations of carbonyl compounds is minimal, in particular, atmospheric concentrations of substituted aromatic aldehydes (efficient sources of aerosol) are close to zero. In spite of a sharp decrease in the intensity of anthropogenic sources, we did not observe a similarly sharp decrease in the concentrations of submicron particles in the air, which means a substantial part of natural sources in the formation of atmospheric organic aerosol.

The processes involved in the formation of atmospheric organic aerosol through photooxidation of various classes of organic compounds were modeled using the model we previously compiled. The model is composed of four main units of elementary stages: 1) hydrocarbon reactions (including methane); 2) reactions of carbonyl compounds-aldehydes and ketones formed during hydrocarbon photooxidation; 3) reactions involving nitrogen oxides and ozone; 4) polymerization and polycondensation of oxidized compounds. The total number of elementary stages in the model was 157 . Initial concentrations of aldehydes were chosen to be equal to the values measured after the introduction of restrictions. Calculations within the

Table 1. Concentrations $\left(\mu \mathrm{g} / \mathrm{m}^{3}\right)$ of carboxylic acids in the air during the six-day experiment

\begin{tabular}{l|l|l|l|l|l|l}
\hline \multirow{2}{*}{\multicolumn{1}{c|}{ Acid }} & \multicolumn{6}{c}{ Day no. } \\
\cline { 2 - 7 } & \multicolumn{1}{c}{1} & \multicolumn{1}{c}{2} & \multicolumn{1}{c}{3} & 4 & 5 & 6 \\
\hline Formic $\mathrm{HCOOH}$ & 1.24 & 1.83 & 1.70 & 2.52 & 2.55 & 2.36 \\
Acetic $\mathrm{CH}_{3} \mathrm{COOH}$ & 2.7 & 3.2 & 3.0 & 0.2 & 0.2 & 0.1 \\
Propionic $\mathrm{C}_{2} \mathrm{H}_{5} \mathrm{COOH}$ & 0.9 & 0.9 & 0.7 & 0.5 & 0.6 & 0.6 \\
Benzoic $\mathrm{C}_{6} \mathrm{H}_{5} \mathrm{COOH}$ & 1.4 & 1.6 & 1.3 & 0.9 & 1.1 & 1.3 \\
\hline
\end{tabular}


model revealed that after the anthropogenic sources were switched off, the largest contribution into the formation of atmospheric organic aerosol was made by the compounds belonging to hydroperoxide class, but the prevailing way of formation of direct precursors of aerosol products in this case is not photopolymerization but photolysis of hydroperoxides, followed by recombination of organic radicals. The second product of the primary photochemical act, namely, the hydroxyl radical, is responsible for hydrogen abstraction from the hydrocarbon fragment, which brings about the possibility of branching the structure of the nucleation precursor.

\section{CONCLUSIONS}

Comparison of the data of atmospheric measurements with the results of calculation within the kinetic model of the formation of aerosol phase precursors shows that the main way of formation of organic aerosol under restriction conditions, which caused a sharp decrease in the intensity of anthropogenic sources, was photodissociation of hydroperoxides, followed by recombination of the formed organic short-lived free radicals. An increase in the ozone concentration in the surface air layer was discovered, which was accompanied by a sharp decrease in the concentration of nitrogen dioxide. The observed changes in the composition of trace gases affected the composition of aerosol products, which was expressed as a decrease in the content of oxygen- and nitrogen-containing groups in the atmospheric organic aerosol.

\section{FUNDING}

The work was supported by the Russian Foundation for Basic Research (project no. 19-43-540 009 r_a).

\section{CONFLICT OF INTERESTS}

The authors declare no conflict of interests.

\section{REFERENCES}

1. A. L. Hansell, K. Adams, and J. Gulliver, "Changes in air quality during COVID-19 'lockdown' in the United Kingdom,” Environ. Pollut. 272, 116011 (2021).

2. M. Wang, S. Lu, M. Shao, L. Zeng, J. Zheng, F. Xie, H. Lin, K. Hu, and X. Lu, "Impact of COVID-19 lockdown on ambient levels and sources of volatile organic compounds (VOCs) in Nanjing, China," Sci. Total Environ. 757, 143823 (2021).

3. S. Zangari, D. T. Hill, A. T. Charette, and J. E. Mirowsky, "Air quality changes in New York city during the COVID-19 pandemic," Sci. Total Environ. 742, 140496 (2020).

4. M. A. Monserrate, M. A. Ruano, and L. S. Alcalde, "Indirect effects of COVID-19 on the environment," Sci. Total Environ. 728, 138813 (2020).

5. G. G. Dultseva, S. N. Dubtsov, and G. I. Skubnevskaya, "Contribution from aldehyde oxidation into the formation of atmospheric organic aerosol," Chem. Sustainable Dev., No. 3, 303-309 (2008).

6. A. V. Keiko, "The dependence of $\mathrm{OH}$ concentration on the intensity of atmosphere surface exchange with nitrogen species," in Proc. 4th Intern. Conf. Chem. Kinet. Gaithersburg, USA, July 14-18, 1997, p. F14.127-128.

7. https://kinetics.nist.gov/. Cited February 26, 2021.

8. J. H. Seinfeld, Atmospehric Chemistry and Physics of Air Pollution (J. Wiley, New York, 2005).

9. S. N. Dubtsov, G. G. Dultseva, E. N. Dultsev, and G. I. Skubnevskaya, "Investigation of aerosol formation during benzaldehyde photolysis," J. Phys. Chem. B 110, 645-649 (2006). 